\title{
Anterior Pituitary Volume in Patients with Transfusion Dependent Anemias: Volumetric Approaches and Relation to Pituitary MRI-R2
}

\author{
Christoph Berliner ${ }^{1}$ Zhiyue J. Wang ${ }^{2}$ Sylvia T. Singer ${ }^{3} \cdot$ Regine Grosse $^{1} \cdot$ Rosalie V. McDonough $^{4}$ Eric Padua $^{3}$. \\ Qing Yuan ${ }^{2}$. Marcela Weyhmiller ${ }^{3}$ Ellen James ${ }^{3}$. Elliott Vichinsky ${ }^{3}$ Gerhard Adam ${ }^{1}$. Jin Yamamura ${ }^{1}$. \\ Peter Bannas ${ }^{1} \cdot$ Roland Fischer $^{1,3} \cdot$ Bjoern P. Schoennagel $^{1}$
}

Received: 17 July 2021 / Accepted: 4 October 2021 / Published online: 28 October 2021

(c) The Author(s) 2021

\begin{abstract}
Purpose Anterior pituitary iron overload and volume shrinkage is common in patients with transfusion-dependent anemia and associated with growth retardation and hypogonadotropic hypogonadism. We investigated the accuracy of different MRI-based pituitary volumetric approaches and the relationship between pituitary volume and MRI-R2, particularly with respect to growth and hypogonadism.

Methods In 43 patients with transfusion-dependent anemia (12-38 years) and 32 healthy controls (12-72 years), anterior pituitary volume was measured by a sagittal T1 GRE 3D sequence at $1.5 \mathrm{~T}$ and analyzed by 3D semi-automated threshold volumetry (3D-volumetry). This reference method was compared with planimetric 2D-volumetry, approximate volume calculations, and pituitary height. Using a multiple SE sequence, pituitary iron as MRI-R2 was assessed by fitting proton signal intensities to echo times. Growth and hypogonadism were obtained from height percentile tables and patients' medical charts. From body surface area and age adjusted anterior pituitary volumes of controls, Z-scores were calculated for all subjects. Separation of controls and patients with respect to $\mathrm{Z}$ and pituitary $\mathrm{R} 2$ was performed by bivariate linear discriminant analysis.

Results Tuned 2D volumes showed highest agreement with reference 3D-volumes (bias $-4.8 \%$; 95\% CI:-8.8\%l-0.7\%). A linear discriminant equation of $Z=-17.8+1.45 \cdot R 2$ revealed optimum threshold sensitivity and specificity of $65 \%$ and $100 \%$ for discrimination of patients from controls, respectively. Of correctly classified patients $71 \%$ and $75 \%$ showed hypogonadism and growth retardation, respectively.

Conclusion Accurate assessment of anterior pituitary size requires 3D or precise 2D volumetry, with shorter analysis time for the latter. Anterior pituitary volume Z-scores and R2 allow for the identification of patients at risk of pituitary dysfunction.
\end{abstract}

Keywords Anterior pituitary gland · Magnetic resonance imaging · Pituitary iron overload · Pituitary R2 - Pituitary volumetry

Availability of Data and Materials The datasets generated and analyzed during the current study are not publicly available due to local restrictions of data protection but are available from the corresponding author on reasonable request.

Bjoern P. Schoennagel

b.schoennagel@uke.de

Department of Diagnostic and Interventional Radiology and Nuclear Medicine, University Medical Center Hamburg-Eppendorf, Martinistr. 52, 20246 Hamburg, Germany
2 Department of Radiology, University of Texas, Southwestern Medical Center, Dallas, TX, USA

3 Departments of Hematology and Radiology, UCSF Benioff Children's Hospital, Oakland, CA, USA

4 Department of Neuroradiology, University Medical Center Hamburg-Eppendorf, Hamburg, Germany 


\section{Introduction}

Endocrine dysfunction from iron accumulation in the anterior pituitary gland is the most common cause of morbidity $(>50 \%)$ in patients with transfusion-dependent anemia (TDA), resulting in hypogonadotropic hypogonadism and growth restriction [1-3]. Pituitary gland size and iron deposition using MRI-R2 methods are validated markers of pituitary function [1, 4-6]; however, pituitary size can be assessed by varying metrics, and the accuracy of these methods has not been validated. Furthermore, the relationship between pituitary size, iron accumulation and gland dysfunction is still poorly understood.

In TDA patients, pituitary height assessed from sagittal MR images is commonly used as a surrogate to volumetric measurements due to its simplicity and speed [5, 7-11]; however, pituitary size and shape vary considerably and assessment of pituitary size is subject to a high degree of imprecision unless true volume is measured [12]. It has been postulated that due to its complex 3-dimensional shape, high-resolution volumetric MRI data would offer more robust metrics of gland health than simple linear height [2]; however, there is a lack of evidence concerning the performance and accuracy of different volumetric approaches, e.g. 2D planimetric models or pituitary height in comparison to the reference standard of pixel-wise 3D analysis.

In addition to providing information on pituitary morphology, MRI can also indirectly measure iron concentration by detecting its paramagnetic properties in pituitary tissue. R2-measurements in the anterior gland have been shown to predict preclinical and biochemical hypogonadism in TDA patients; however, there is paucity of data concerning the relation of pituitary volume and pituitary iron deposition [1, 13, 14]. Similarly, there is limited evidence regarding the relationship of clinical manifestations of pituitary dysfunction (e.g. growth retardation) with pituitary volume and iron accumulation using MRI-R2 relaxometry $[15,16]$.

The aim of this retrospective study was to determine the accuracy of different approaches for the assessment of anterior pituitary size and the relationship between pituitary size and MRI-R2 relaxometry, particularly with respect to growth retardation and hypogonadism, in patients with TDA.

\section{Methods}

\section{Study Population}

A total of 43 consecutive TDA patients ( 22 females; mean age 22 years, range: $10-41$ years), regularly receiving blood transfusions and treated with iron chelation agents were included in this retrospective study. The underlying diseases were transfusion dependent thalassemia $(n=25)$, Diamond Blackfan anemia $(n=7)$, sickle cell disease $(n=6)$, and rare forms of anemia (sideroblastic, congenital dyserythropoietic and Fanconi anemia). We included 32 controls (19 males; mean age 38 years, range: $12-86$ years) without clinical or anamnestic signs of endocrinopathy or hypothalamic region pathology (Table 1) and 13 children (age $\leq 18$ years) were included in the patient group and 4 children in the control group.

Body height and weight was measured for each individual at the time of MRI assessment to calculate body surface area (BSA) and growth percentiles. The latter was calculated from height, gender, age, and ethnicity using the World Health Organization (WHO) (https://www. omnicalculator.com/health/child-height-percentile) or the Centers for Disease Control and Prevention (CDC) (https:// tall.life/height-percentile-calculator-age-country) standard.

3-dimensional volumes (V3D) and pituitary R2 were available for all patients and assessed within 0-3 months

Table 1 Characteristics of transfusion dependent anemia (TDA) patients and controls: median and interquartile range (IQR) of age, body surface area (BSA), pituitary height, 3D volume (V3D) and 2D volume (V2D $\left.\mathrm{D}_{\text {tuned }}\right)$, and body height percentiles; Mann-Whitney U-test between groups, and Spearman rank correlation ( $\left.\mathrm{R}_{\mathrm{S}}, p\right)$ of pituitary $\mathrm{R} 2$ with other parameters (R2xParam.)

\begin{tabular}{|c|c|c|c|c|c|c|c|c|}
\hline \multirow[t]{2}{*}{ Parameter } & \multicolumn{3}{|c|}{ TDA patients } & \multicolumn{3}{|c|}{ Controls } & \multirow{2}{*}{$\begin{array}{l}\mathrm{U} \text {-test } \\
p\end{array}$} & \multirow{2}{*}{$\begin{array}{l}\mathrm{R} 2 \text { xparam } \\
R_{S}, p\end{array}$} \\
\hline & $n$ & Median & $I Q R$ & $n$ & Median & $I Q R$ & & \\
\hline Children ( $\leq 18$ years) & 13 & - & - & 4 & - & - & - & - \\
\hline Age (years) & 43 & 20 & 8 & 32 & 35 & 25 & $<10^{-5}$ & $0.10,0.4$ \\
\hline $\operatorname{BSA}\left(\mathbf{m}^{2}\right)$ & 43 & 1.5 & 0.2 & 32 & 1.8 & 0.3 & $<10^{-6}$ & $-0.18,0.2$ \\
\hline Pituitary R2 $\left(\mathrm{s}^{-1}\right)$ & 43 & 13 & 5 & 22 & 10 & 1 & $<10^{-4}$ & $1.0,0$ \\
\hline Pituitary height (mm) & 43 & 5.5 & 1.4 & 32 & 7.0 & 1.0 & $<10^{-6}$ & $-0.53,5 \cdot 10^{-6}$ \\
\hline V3D $\left(\mathbf{m m}^{3}\right)$ & 43 & 349 & 197 & 30 & 527 & 124 & $<10^{-3}$ & $-0.55,3 \cdot 10^{-6}$ \\
\hline V3D $D_{\text {males }}\left(\mathrm{mm}^{3}\right)$ & 21 & 385 & 158 & 18 & 494 & 128 & $2 \cdot 10^{-2}$ & $-0.47,6 \cdot 10^{-3}$ \\
\hline V3D $_{\text {females }}\left(\mathbf{m m}^{\mathbf{3}}\right)$ & 22 & 319 & 211 & 12 & 571 & 115 & $<10^{-2}$ & $-0.65,10^{-4}$ \\
\hline V2D tuned $\left(\mathrm{mm}^{3}\right)$ & 33 & 346 & 141 & 25 & 541 & 108 & $<10^{-3}$ & $-0.45,10^{-3}$ \\
\hline Body height (\%) & 43 & 21 & 40 & 32 & 70 & 32 & $<10^{-5}$ & $-0.19,0.1$ \\
\hline
\end{tabular}


(median: same day) of the MRI measurement of the pituitary gland (Table 1). Diagnosis of hypogonadism was obtained from medical charts. Diagnostic criteria involved lack of secondary puberty, amenorrhea in females or need of testosterone replacement in males. Females or males between 10-14y with constantly low gonadotropin and estradiol/testosterone levels were also diagnosed for hypogonadism.

Patients and controls were measured between 2006-2017 at the radiology departments of two university medical centers in Europe $(n=40)$ and the USA $(n=35)$. All procedures were approved by the institutional committees on human research at both centers. Informed written consent was obtained from all individuals.

\section{MR Imaging Protocol}

MRI of the pituitary gland was performed using an 8-element head coil on two different 1.5T MRI systems (Symphony ${ }^{\circledR}$, Siemens Healthcare, Erlangen, Germany; Intera ${ }^{\circledR}$, Philips Healthcare, Best, The Netherlands).

For Siemens/Philips systems and volume assessment, a gradient-recalled echo $\mathrm{T} 1$ weighted 3D sequence in sagittal orientation was applied with $1 \mathrm{~mm}$ isotropic voxels $\left(\mathrm{TR}=17 / 22 \mathrm{~ms}, \mathrm{TE}=3.7 / 4.6 \mathrm{~ms}, \mathrm{FA}=12^{\circ} / 30^{\circ}\right.$, matrix $=256 \times 256$, pixel size $1.0 \times 1.0 \mathrm{~mm}^{2}, 100$ slices, slice thickness $=1 \mathrm{~mm}$, gap $=0 \mathrm{~mm}$, bandwidth $=210 / 47 \mathrm{~Hz} / p \mathrm{x})$. Pituitary R2 was assessed using a multi-echo spin-echo sequence in sagittal orientation (Siemens/Philips: $T R=$
$2500 \mathrm{~ms}, \quad \mathrm{TE} 1=15 \mathrm{~ms}, \quad \Delta \mathrm{TE}=15 \mathrm{~ms}$, echo train length 8 , matrix $=256 \times 256$, pixel size $0.78 \times 0.78 \mathrm{~mm}^{2}$, slices $=11$, slice thickness $=3 \mathrm{~mm}$, gap $=0.3 \mathrm{~mm}$, bandwidth $=95 / 120 \mathrm{~Hz} / p x$ ). Total data acquisition time was approximately $30 \mathrm{~min}$. The MRI protocol was implemented by one experienced radiologist (Z.J.W.) at both institutions.

A monoexponential function for proton signal intensities and echo time (including all 8 echoes) was used for determination of the relaxation rate $\mathrm{R} 2$ with no signal level offset using an in-house software (IDL 7.0). The anterior pituitary ROI was manually drawn along the boundary of the gland from 3-4 slices. Agreement between R2 from pixelwise and ROI-wise analysis was $-0.07 \pm 0.04 \mathrm{~s}^{-1}$ (95\% CI: $\left.-0.15 \mathrm{~s}^{-1}-0.02 \mathrm{~s}^{-1}\right)$.

\section{Pituitary Volumetry}

\section{Semi-automated Threshold 3D Volumetry (V3D)}

The approach combined two methods for determination of the boundary of the anterior pituitary gland. Firstly, the ROI information was provided in two slice orientations (sagittal and axial reconstructions). Manual tracing was performed using multiple sagittal and axial planes. The computer routine combines the tracing to obtain a 3D ROI. This twoplane tracing approach was used because different boundaries were best visualized on different orientations. For example, the boundary of the left and right side of the pituitary gland from the adjacent cavernous sinus was easy

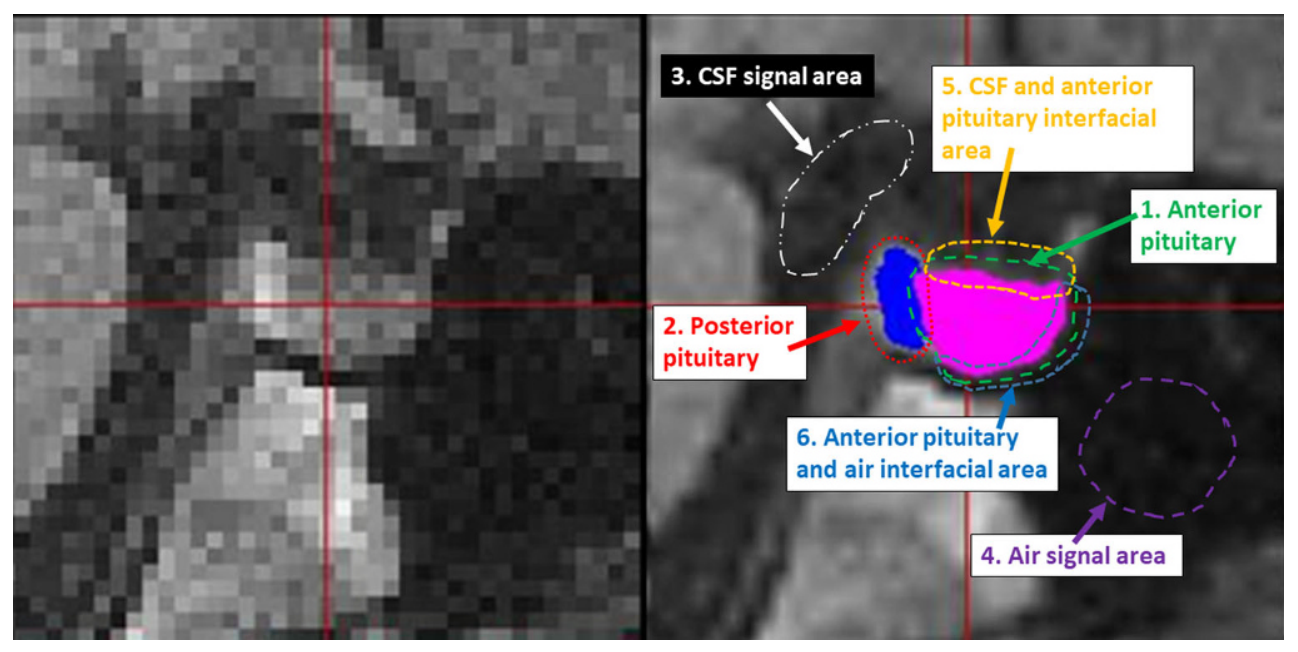

Fig. 1 Demonstration of semi-automatic threshold in V3D anterior pituitary volumetry. The left shows a T1-weighted mid-sagittal MR image of the pituitary gland. On the right, the image is 6-fold interpolated, and manually drawn ROIs used for generating the anterior pituitary mask are shown. ROIs of anterior (1) and posterior (2) pituitary were sufficiently eroded to remove the exterior signal background before the interior signal was averaged. The signals for CSF (ROI 3), and air space (ROI 4) were directly averaged. The boundary between the anterior pituitary and CSF was determined in ROI 5 by setting the threshold to the average of CSF mean value and anterior pituitary mean value. The boundary between the anterior pituitary and air space was determined in ROI 6 by setting the threshold to the average of air space mean value and anterior pituitary mean value. The boundary between the anterior and posterior pituitary was determined inside the overlapping region of ROIs 1 and 2, with the threshold equal to the average signal intensity of the two glands. The solid pink and blue areas represent the final masks used for anterior and posterior pituitary volume calculation 
to visualize on the axial plane but difficult on the sagittal plane. Second, the boundaries of the anterior and posterior pituitary glands were determined by setting thresholds in interfacial areas with surrounding structures (Fig. 1). Anterior pituitary volumes were then obtained. One data set can be processed in approximately $20 \mathrm{~min}$. Details of the software have been previously described [17].

\section{Planimetric 2D Volumetry (V2D)}

MRI 3D data sets of the pituitary were imported into an external analysis software (Volume Viewer, GE-Healthcare, Chicago, IL, USA). The external contours of the anterior pituitary gland were manually delineated in all axial slices covering the anterior pituitary ( $\leq 10$ slices). The posterior pituitary gland was differentiated from the anterior gland by its brighter signal intensity. The volume was obtained by adding the areas of each slice. Volumes were independently assessed by two operators (CB and BS) to determine

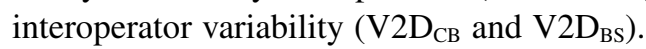

In a more complex step, delineation of pituitary contours was controlled and eventually corrected in all 3 planes (axial, coronal, sagittal) resulting in a fine-tuned $2 \mathrm{D}$ volume (V2D $\mathrm{D}_{\text {tuned }}$ ). One data set was processed in about $5-10 \mathrm{~min}$.

\section{Approximate Volumetry $\left(\mathrm{V}_{\mathrm{ROI}}, \mathrm{V}_{\mathrm{EIII}}\right.$, and $\left.\mathrm{V}_{\mathrm{AL}}\right)$}

Approximate assessment of anterior pituitary volumes was based on the classical formula for the volume of an ellipsoid, $\mathrm{V}=4 \pi / 3 \cdot \mathrm{a} \cdot \mathrm{b} \cdot \mathrm{c}$, with axes $\mathrm{a}, \mathrm{b}$, and $\mathrm{c}$. The finetuned $\mathrm{V} 2 \mathrm{D}_{\text {tuned }}$ procedure also allowed the determination of several pituitary ROIs and diameters in different planes: $\mathrm{ROI}_{\text {trans, }}, \mathrm{ROI}_{\text {cor }}$, and left-to-right $\left(\mathrm{d}_{\mathrm{lr}}\right)$, anterior-to-posterior $\left(\mathrm{d}_{\mathrm{ap}}\right)$, and feet-to-head $\left(\mathrm{d}_{\mathrm{fh}}\right)$ diameter. The following approximate volumes of the anterior pituitary gland were calculated as

$\mathrm{V}_{\mathrm{ROI}}=8 / 3 \pi \cdot \mathrm{ROI}_{\text {trans }} \cdot \mathrm{ROI}_{\mathrm{cor}} / \mathrm{d}_{\mathrm{lr}}$,

$\mathrm{V}_{\text {Ell }}=\pi / 6 \cdot d_{\mathrm{ap}} \cdot \mathrm{d}_{\mathrm{fh}} \cdot \mathrm{d}_{\mathrm{lr}}$,

and by the area-length method $\mathrm{V}_{\mathrm{AL}}=2 / 3 \cdot \mathrm{ROI}_{\text {trans }} / \mathrm{d}_{\mathrm{fh}}$.

The most straightforward information about the size of the anterior pituitary is the maximum pituitary height measured in mid-sagittal images as previously described [18].

\section{Statistics}

All values are presented as median values with interquartile ranges (IQR). Variables between groups were tested for significance using the nonparametric Wilcoxon-MannWhitney U-test. $P$-values $<0.05$ were considered statistically significant. Pituitary R2 was compared with other parameters using Spearman-rank correlation $\left(\mathrm{r}_{\mathrm{s}}\right)$. Comparison of volumetric approaches was performed by relative difference analysis versus the reference method of V3D. The interoperator variability was assessed by Bland-Altman analysis.

Multiple regression analysis (forward and backward stepwise) was used for prediction, characterized by the coefficient of determination $\left(\mathrm{r}^{2}\right)$. Normal BSA-adjusted pituitary $3 \mathrm{D}$ volumes $\left(\mathrm{V} 3 \mathrm{D}_{\mathrm{i}}\right)$ were calculated from controls as a function of age, subtracted from measured $V 3 \mathrm{D}_{\mathrm{i}}$, and expressed as Z-values. Separation of controls and TDA patients with respect to $\mathrm{Z}$ and pituitary $\mathrm{R} 2$ was performed by bivariate linear discriminant analysis with a priori equal group size, which brings different patient $(n=43)$ and control numbers $(n=32)$ to the same level (STATISTICA 6.1, Stat Soft. Inc., Tulsa, OK, USA).

\section{Results}

Transfusion dependent anemia (TDA) patients were significantly younger, had significantly lower BSA, pituitary height, and volume (V3D), but higher anterior pituitary R2 values compared to controls $\left(p<10^{-4}\right)$. Significant correlations with pituitary R2 were found for pituitary height and volume (Table 1).

\section{Pituitary Volume Data Analysis}

Pituitary height was assessed in all controls $(n=32)$ and TDA patients $(n=43)$. Pituitary planimetry (V2D $\left.\mathrm{D}_{\text {tuned }}\right)$ and volumetric estimations by $\mathrm{V}_{\mathrm{RO}}, \mathrm{V}_{\mathrm{Ell}}$, and $\mathrm{V}_{\mathrm{AL}}$ were assessed in 58 of 75 patients and controls (77\%). Comparison of in-

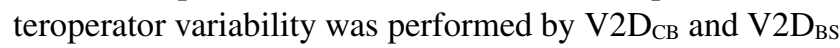
and in 48 subjects. Reference 3-dimensional volume (V3D) was assessed in 42 patients and 30 controls $(n=72,96 \%)$.

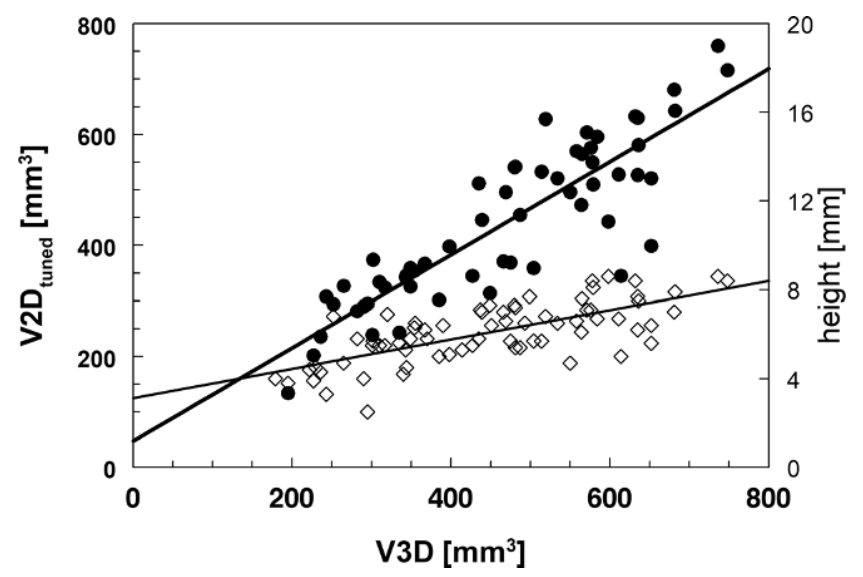

Fig. 2 Significant correlation between pituitary volumetric methods

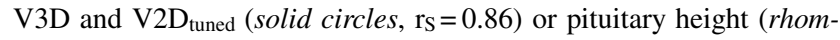
boids, $\mathrm{rs}=0.64$, right scale) in all subjects 
Table 2 Pituitary volumes assessed by MRI: comparison of reference V3D and other approximate volumetric methods $\left(p<10^{-4}\right)$, numbers indicate relative differences with $\pm 95 \%$ lower and upper limit of agreement (LoA, UpA)

\begin{tabular}{|c|c|c|c|c|c|c|c|}
\hline Methods & $n$ & $\mathrm{R}_{\mathrm{S}}{ }^{\mathrm{a}}$ & $\begin{array}{l}\text { Bias } \\
(\%)\end{array}$ & $\begin{array}{l} \pm \mathrm{SE} \\
(\%)\end{array}$ & $\begin{array}{l}-95 \% \text { LoA } \\
(\%)\end{array}$ & $\begin{array}{l}+95 \% \text { UpA } \\
(\%)\end{array}$ & $\begin{array}{l} \pm \mathrm{SE} \\
(\%)\end{array}$ \\
\hline V3DxV2D tuned & 58 & 0.86 & -4.8 & 2.0 & -35.0 & 25.4 & 3.5 \\
\hline V3DxV2D & 49 & 0.72 & -14.9 & 3.0 & -56.6 & 26.8 & 5.2 \\
\hline V3DxV2D & 48 & 0.73 & -13.3 & 3.0 & -54.4 & 27.9 & 5.2 \\
\hline V3DxV & 58 & 0.69 & -24.3 & 2.9 & -68.2 & 19.5 & 5.1 \\
\hline V3DxVEII & 58 & 0.63 & -21.0 & 3.2 & -69.5 & 27.5 & 5.6 \\
\hline V3DxV $V_{A L}$ & 58 & 0.75 & -18.7 & 2.8 & -60.6 & 23.4 & 4.8 \\
\hline V2D $_{C B} \times V 2 D_{B S}{ }^{b}$ & 48 & 0.95 & 2.6 & 1.7 & -20.8 & 26.0 & 3.0 \\
\hline
\end{tabular}

$V 3 D$ semi-automated threshold $3 \mathrm{D}$ volumetry, $V 2 D_{\text {tuned }}$ planimetric $2 \mathrm{D}$ volumetry with corrections, $V 2 D_{C B}$ planimetric $2 \mathrm{D}$ volumetry by operator $\mathrm{CB}, V 2 D_{B S}$ planimetric $2 \mathrm{D}$ volumetry by operator BS, $V_{R O I}$ approximate volumetry by region of interest, $V_{E l l}$ approximate volumetry by ellipsoid model, $V_{A L}$ approximate volumetry by area-length model

${ }^{\text {a }}$ Spearman rank correlation coefficient

${ }^{\mathrm{b}}$ Bland-Altman test

The missing V3D volumes were due to artifacts or incomplete 3D-data sets.

V3D as the volumetric standard of reference was compared to all other pituitary measures by Pearson correlation and relative difference (Altman-Bland) tests. In Fig. 2, exemplary significant correlations (both $p<0.0001$ ) of the anterior pituitary volumes V3D with V2 $\mathrm{D}_{\text {tuned }}\left(\mathrm{R}_{S}=0.86\right)$ and pituitary height $\left(R_{S}=0.64\right)$ are shown.

Relative differences between the reference volume V3D and other volumetric estimators are shown in Table 2. The 2-dimensional method V2D $\mathrm{D}_{\text {tuned }}$ showed the highest agreement with the reference volume, with a mean deviation of $-4.8 \%$ (95\%CI: $-8.8 \%-0.7 \%$ ). Comparison of $\mathrm{V}_{2} \mathrm{D}_{\mathrm{CB}}$ with $\mathrm{V}_{\mathrm{ROI}}$ and $\mathrm{V}_{\mathrm{Ell}}$ revealed mean deviations of $-9.0 \%$ and $-10.0 \%$, respectively (data not shown).

Interoperator agreement for V2D between the two oper-

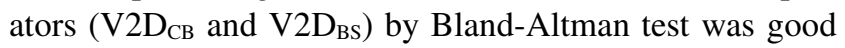
(bias $2.6 \% \pm$ SE $1.7 \%$ ) (Table 2).

\section{BSA-adjusted Pituitary Volume and Age in Controls}

For controls, multiple regression of pituitary volume (V3D) with age, BSA, and R2 resulted in significant contributions only from age $(p=0.01)$, in contrast to the negative univariate regression with age $\mathrm{V} 3 \mathrm{D}\left(\mathrm{mm}^{3}\right)=604-2.3 \cdot$ age $\left(\mathrm{r}^{2}=0.11\right.$, $p=0.04$ ).

This relationship with age significantly improved for

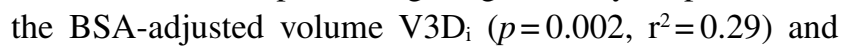
resulted in a linear relationship of $\mathrm{V}_{3} \mathrm{D}_{\mathrm{i}}=\mathrm{a} 0+\mathrm{a} 1 \cdot$ age with $\mathrm{a} 0=368 \pm 25 \mathrm{~mm}^{3} / \mathrm{m}^{2}, \quad \mathrm{a} 1=-2.16 \pm 0.61 \mathrm{~mm}^{3} / \mathrm{m}^{2} /$ year, and a standard error of estimate (SEE) $=57 \mathrm{~mm}^{3} / \mathrm{m}^{2}$ (Fig. 3). The obtained equation allows calculation of anterior pituitary reference volumes as function of age $\left(\mathrm{V} 3 \mathrm{D}_{\mathrm{i}}(\right.$ age $\left.)\right)$ from controls and thus Z-scores for all subject volumes $\left(\mathrm{V} \mathrm{D}_{\mathrm{i}}\right)$ by $\mathrm{Z}=\left(\mathrm{V} 3 \mathrm{D}_{\mathrm{i}}-\mathrm{V} 3 \mathrm{D}_{\mathrm{i}}(\right.$ age $\left.)\right) / \mathrm{SEE}$.

\section{Discriminant Analysis of Patients from Controls by Z-scores and $\mathbf{R 2}$}

Since age, R2, and BSA are more strongly associated with V3D of patients compared to controls, multiple regression analysis was applied to all subjects resulting in significant contributions from BSA $(p=0.0003), \mathrm{R} 2(p=0.0005)$, and age $(p=0.03)$. R2 $(p=0.0002)$ and age $(p=0.04)$ were sig-

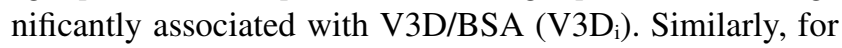
BSA adjusted pituitary height, we obtained significant associations from R2 $(p=0.001)$ and age $(p=0.03)$, in contrast to V2D/BSA, where only R2 contributed significantly $(p=0.01)$.

Using age and BSA adjusted V3D Z-scores Z(V3D age) for pituitary volume in patients $(n=43)$ and controls $(n=19)$ allowed separation of the two groups by bivariate discriminant analysis $(\mathrm{Z}, \mathrm{R} 2)$ along a linear discriminant (cut-off) line of $Z=-17.8+1.45 \cdot R 2$ (Fig. 4). A sensitivity and specificity of 65\% (28/43) and 100\% (19/19) was obtained for correctly classifying patients and controls, respectively. Univariate discriminant analysis resulted in thresholds of $\mathrm{Z}\left(\mathrm{V} 3 \mathrm{D}_{\mathrm{i}}\right.$, age $)=-0.54$ or $\mathrm{R} 2=11.9 \mathrm{~s}^{-1}$ with specificities of $76 \%$ or $100 \%$, respectively, and similar sensitivities $(70 \%$ or $67 \%)$. For bivariate age and BSA adjusted V2D Z-scores $Z\left(V 2 D_{i}\right.$, age) versus $R 2$, discriminant analysis in only 42 patients and controls showed a sensitivity of $61 \%$ and a specificity of $93 \%$ (data not shown).

Growth was assessed as height percentiles with median values (IQR) for patients and controls of 21\% (9-49\%) and $70 \%$ (53-85\%), respectively (Table 1). Univariate discriminant analysis based on growth significantly separated patients and controls at $47.2 \%$ (sensitivity $70 \%$ and specificity $78 \%$ ). In the 28 patients correctly classified by the linear discriminant equation of Fig. 4, growth was reduced in 21 $(\mathrm{IQR}=9 \%-29 \%)$ of them with a sensitivity of $75 \%$, while 


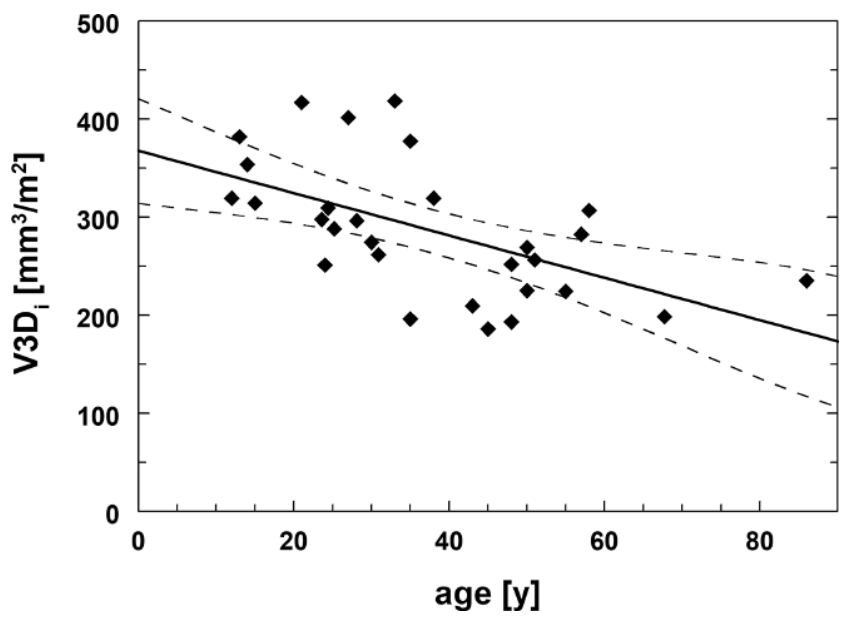

Fig. 3 Relationship between BSA adjusted V3D $\left(=V 3 \mathrm{D}_{\mathrm{i}}\right)$ and age in controls (dashed lines indicate $95 \%$ confidence interval, $\mathrm{r}^{2}=0.29$ )

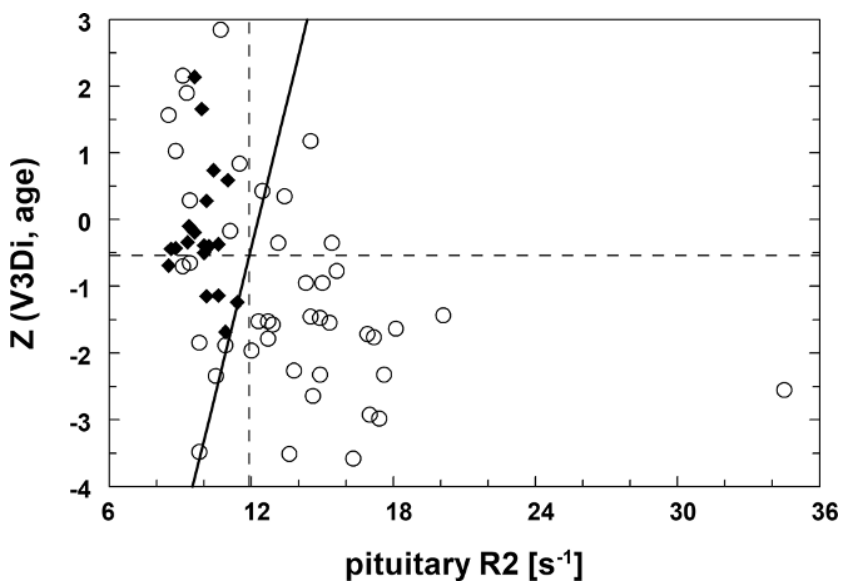

Fig. 4 Z-values calculated from BSA and age adjusted pituitary 3Dvolumes $\left(\mathrm{Z}\left(\mathrm{V} 3 \mathrm{D}_{\mathrm{i}}\right.\right.$, age $\left.)\right)$ are associated with pituitary relaxation rate $\mathrm{R} 2$. A linear discriminant function (solid line, $p<0.0016$ ) separates controls (rhomboids) from transfusion dependent patients (circles). Univariate thresholds for $\mathrm{R} 2$ and $\mathrm{Z}$ are indicated by dashed lines at $11.9 \mathrm{~s}^{-1}$ and -0.54 , respectively

7 patients (IQR $=57 \%-80 \%$ ) were in the range of controls (IQR $=54 \%-82 \%$ ) (data not shown).

Hypogonadism was reported in $31 / 42$ patients. Using a bivariate discriminant analysis $\left(\mathrm{Z}\left(\mathrm{V} 3 \mathrm{D}_{\mathrm{i}}\right.\right.$, age $\left.), \mathrm{R} 2\right)$ along a linear discriminant (cut-off) line of $\mathrm{Z}=-2.11+0.087 \cdot \mathrm{R} 2$ allowed separation of patients with and without hypogonadism (Fig. 5). A sensitivity of $74 \%$ was obtained for correctly classifying patients with hypogonadism. The same sensitivity could be achieved for V3D/BSA alone at a threshold of $270 \mathrm{~mm}^{3} / \mathrm{m}^{2}$, while $\mathrm{R} 2$ alone was relatively insensitive for hypogonadism (48\%). For univariate V2D Z-scores $\mathrm{Z}\left(\mathrm{V} 2 \mathrm{D}_{\mathrm{i}}\right.$, age), a sensitivity of $73 \%$ at a threshold of $275 \mathrm{~mm}^{3} / \mathrm{m}^{2}$ was calculated for $26 / 33$ patients with hypogonadism.

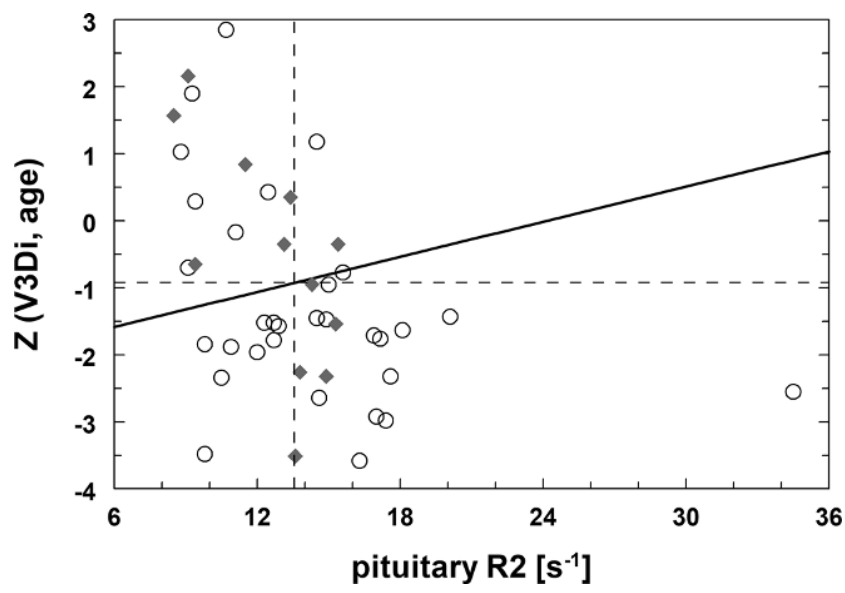

Fig. 5 Z-values calculated from BSA and age adjusted pituitary 3Dvolumes $\left(\mathrm{Z}\left(\mathrm{V} 3 \mathrm{D}_{\mathrm{i}}\right.\right.$, age $\left.)\right)$ are associated with pituitary relaxation rate $\mathrm{R} 2$. A linear discriminant function (solid line, $p<0.0016$ ) separates patients with hypogonadism (circles) and without hypogonadism (rhomboids). Univariate thresholds for $\mathrm{R} 2$ and $\mathrm{Z}$ are indicated by dashed lines at $13.6 \mathrm{~s}^{-1}$ and -0.92 , respectively

\section{Discussion}

This MRI study in patients with TDA evaluated the accuracy of different geometric approaches for assessing the volume of the anterior pituitary gland. It also investigated the association of pituitary size, growth retardation, and hypogonadism, as an indicator of pituitary dysfunction, with iron levels in the pituitary gland using MRI-R2 relaxometry.

Compared to the reference standard of semi-automated threshold-dependent V3D, V2D planimetry revealed the highest agreement and was superior to approximated volumetry $\left(\mathrm{V}_{\mathrm{ROI}}, \mathrm{V}_{\mathrm{Ell}}\right.$, and $\left.\mathrm{V}_{\mathrm{AL}}\right)$ and pituitary height. Z-scores of pituitary volume were associated with $\mathrm{R} 2$ rates and revealed threshold-derived specificities of $76 \%$ and $100 \%$ for both parameters to discriminate patients from controls, respectively. In $75 \%$ and $71 \%$ of patients correctly classified by volume Z-scores and $\mathrm{R} 2$ rates, growth restriction (defined below the 47 th percentile) and hypogonadism was present, respectively.

As pituitary size is an independent predictor of preclinical hypogonadism in adult patients, reliable measurement of the anterior pituitary volume is critical [1]. Few studies have performed true planimetry (V2D) $[12,19,20]$ or indirect volume estimations $\left(\mathrm{V}_{\mathrm{ROI}}\right.$ and $\left.\mathrm{V}_{\mathrm{ell}}\right)[12,21,22]$ and, to our knowledge, even fewer employed a more sophisticated semi-automated V3D approach [23]. In this study, volumetric comparisons using V3D as the reference method revealed highest accuracy for $\mathrm{V} 2 \mathrm{D}$ planimetry ( $\left.\mathrm{V}_{2} \mathrm{D}_{\text {tuned }}\right)$ with a mean deviation of only $-5 \%$. Conversely, indirect volume estimates $\left(\mathrm{V}_{\mathrm{ROI}}, \mathrm{V}_{\mathrm{Ell}}, \mathrm{V}_{\mathrm{AL}}\right)$ revealed significant bias of up to $-24 \%$ and appear insufficient to reflect true pituitary volumes. Our results confirm the hypothesis that size assessment is subject to a high degree of imprecision un- 
less the true volume is measured [12]. Considering the long post-processing times of around $20 \mathrm{~min}$ and the dedicated expertise and software required for $\mathrm{V} 3 \mathrm{D}$, we recommend

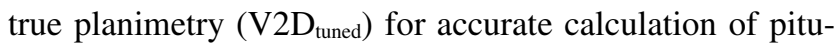
itary gland volumes. The required 3D image data acquisitions and an approximated post-processing time of $5 \mathrm{~min}$ are assumed worthwhile, considering the merit of precise volumetric results. The good interoperator agreement for V2D (bias below 3\%) emphasizes its clinical utility in daily routine.

BSA-adjusted anterior pituitary volumes of healthy controls were in agreement with reported normal values [20] considering the older age of our controls. There is a wellknown relation of anterior pituitary volume with age, revealing a typical increase before and during puberty [6, 12], followed by a plateau phase or slight decline of volume starting in the 3rd decade of life [8, 20,21]. Our study using V3D also revealed a decrease in anterior pituitary volume with age and encompassed a broader age spectrum than former studies which focused on the first and second decades of life $[10,12,19,24]$. Our study revealed lower pituitary volumes $\left(\right.$ mean $\left.=516 \mathrm{~mm}^{3}\right)$ for controls than a study in 94 women (age 18-90 years) [25]; however, the inverse relationship with age could be confirmed if volumes were adjusted for these lower values within limits.

Anterior pituitary volume Z-scores (adjusted for BSA and age) were associated with pituitary $\mathrm{R} 2$ rates. Combined volume Z-scores and R2 allowed reliable discrimination of patients from controls with a sensitivity and specificity of $65 \%$ and $100 \%$, respectively. From univariate analysis, R2 was superior to discriminate patients from controls than volumetric Z-scores. Pituitary size can be affected by different factors, but in patients with TDA pituitary shrinkage is primarily thought to originate from iron deposition $[1,2$, $7,13]$. The fact that volumetric Z-scores were significantly dependent on R2 supports the idea of pituitary iron deposition to be the causative mechanism for developing pituitary volume loss.

Hypogonadism and growth retardation are frequent endocrine complications associated with the anterior pituitary gland in TDA patients, with a reported prevalence of $50-80 \%$ [26, 27]; however, there are only few studies investigating the association of pituitary iron and endocrine function. In our cohort of TDA patients, growth restriction and hypogonadism were clearly linked to pituitary R2 and volume loss. In patients who were correctly identified by abnormal pituitary volume Z-scores and R2, growth retardation defined by the 47th percentile and hypogonadism were a frequent finding (75\% and $71 \%$ ). This is comparable to observations where pituitary size and excess iron were independently associated with hypogonadism $[1,4$, $5,13]$. In addition, our results showed that precise volume assessment seems to be more important than $\mathrm{R} 2$ to predict hypogonadism.

Although R2 is an independent biomarker of pituitary function, the causal relationship with pituitary size is not fully understood and studies to analyze this relation are rare $[1,4]$. This study supports the finding that pituitary iron deposition reflected by R2 leads to pituitary volume loss and subsequent gland dysfunction, as corroborated by the high rate of growth retardation in our patient group.

The etiology of pituitary dysfunction is complex but is mainly attributed to excess iron depositions $[13,15]$, with the anterior lobe being particularly prone to the toxic effects of iron overload [28]; however, factors predicting the development of pituitary iron overload are not well understood $[1,11]$.

A potential limitation of this study is the lack of endocrine measures (e.g. hormone stimulating tests); however, these tests have been described as being imprecise and suffer from poor reproducibility [29]. We also did not investigate the impact of estrogen/testosterone on pituitary size since many patients were under replacement therapy. Although MR imagers from two manufacturers were used, no significant differences were observed between Siemens/Philips for R2 $(n=34 / 31, p=0.4)$ or V3D $(n=34 / 39, p=0.2)$.

Other limitations like missing age and gender matched controls were due to the retrospective character of the study; however, the utilization of Z-scores usually compensates for this drawback. With respect to Z-scores a more uniform stratification of age would have been beneficial.

\section{Conclusion}

Accurate assessment of pituitary size requires true V3D or

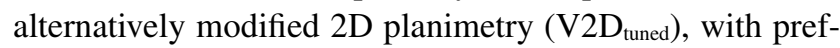
erence of the latter due to shorter analysis time and no need for specialized software. Pituitary volume and R2 allow the discrimination of TDA patients from healthy individuals. Abnormal values are frequently associated with growth retardation, and hypogonadism may be preferentially predicted by volume shrinkage. In the future, assessment of pituitary volume and R2 may help to detect gland damage before endocrine dysfunction becomes overt. Further research is necessary to better understand the relation of pituitary volume, $\mathrm{R} 2$ and endocrine dysfunction relative to age.

Funding This study received funding from the Palliative Care Research Cooperative.

Funding Open Access funding enabled and organized by Projekt DEAL. 


\section{Declarations}

Conflict of interest C. Berliner, Z.J. Wang, S.T. Singer, R. Grosse, R.V. McDonough, E. Padua, Q. Yuan, M. Weyhmiller, E. James, E. Vichinsky, G. Adam, J. Yamamura, P. Bannas, R. Fischer and B.P. Schoennagel declare that they have no competing interests.

Ethical standards Ethical approval was waived by the local institutional committee on human research in view of the retrospective nature of the study. Consent to participate: all participants of the study gave their written informed consent prior to examination. Consent for publication: all participants gave approval for their data to be published.

Open Access This article is licensed under a Creative Commons Attribution 4.0 International License, which permits use, sharing, adaptation, distribution and reproduction in any medium or format, as long as you give appropriate credit to the original author(s) and the source, provide a link to the Creative Commons licence, and indicate if changes were made. The images or other third party material in this article are included in the article's Creative Commons licence, unless indicated otherwise in a credit line to the material. If material is not included in the article's Creative Commons licence and your intended use is not permitted by statutory regulation or exceeds the permitted use, you will need to obtain permission directly from the copyright holder. To view a copy of this licence, visit http://creativecommons.org/licenses/by/4. $0 /$.

\section{References}

1. Noetzli LJ, Panigrahy A, Mittelman SD, Hyderi A, Dongelyan A, Coates TD, Wood JC. Pituitary iron and volume predict hypogonadism in transfusional iron overload. Am J Hematol. 2012;87:167-71.

2. Wood JC, Noetzl L, Hyderi A, Joukar M, Coates T, Mittelman S. Predicting pituitary iron and endocrine dysfunction. Ann N Y Acad Sci. 2010;1202:123-8.

3. Borgna-Pignatti C, Rugolotto S, De Stefano P, Zhao H, Cappellini MD, Del Vecchio GC, Romeo MA, Forni GL, Gamberini MR, Ghilardi R, Piga A, Cnaan A. Survival and complications in patients with thalassemia major treated with transfusion and deferoxamine. Haematologica. 2004;89:1187-93.

4. Wood JC. Estimating tissue iron burden: current status and future prospects. Br J Haematol. 2015;170:15-28.

5. Argyropoulou MI, Kiortsis DN, Metafratzi Z, Bitsis S, Tsatoulis A, Efremidis SC. Pituitary gland height evaluated by MR in patients with beta-thalassemia major: a marker of pituitary gland function. Neuroradiology. 2001;43:1056-8.

6. Wong AP, Pipitone J, Park MTM, Dickie EW, Leonard G, Perron M, Pike BG, Richer L, Veillette S, Chakravarty MM, Pausova $\mathrm{Z}$, Paus T. Estimating volumes of the pituitary gland from T1weighted magnetic-resonance images: effects of age, puberty, testosterone, and estradiol. Neuroimage. 2014;94:216-21.

7. Argyropoulou MI, Kiortsis DN, Astrakas L, Metafratzi Z, Chalissos N, Efremidis SC. Liver, bone marrow, pancreas and pituitary gland iron overload in young and adult thalassemic patients: a T2 relaxometry study. Eur Radiol. 2007;17:3025-30.

8. Mohieldin AE, Mohamed DA, Mahmoud MZ, Fagiri MA, Abukonna A. Effect of age and gender variation in normal pituitary gland height using magnetic resonanceimaging. Br J Med Med Res. 2016;18:1-8.

9. Bozdağ M, Bayraktaroğlu S, Aydınok Y, Çallı MC. MRI assessment of pituitary iron accumulation by using pituitary-R2 in $\beta$-thalassemia patients. Acta Radiol. 2018;59:732-9.

10. Tsunoda A, Okuda O, Sato K. MR height of the pituitary gland as a function of age and sex: especially physiological hypertro- phy in adolescence and in climacterium. AJNR Am J Neuroradiol. 1997;18:551-4.

11. Çetinçakmak MG, Hattapoğlu S, Menzilcioğlu S, Alan B, Uluca Ü, Uçar A, Söker M, Bilici A. MRI-based evaluation of the factors leading to pituitary iron overload in patients with thalassemia major. J Neuroradiol. 2016;43:297-302.

12. Fink AM, Vidmar S, Kumbla S, Pedreira CC, Kanumakala S, Williams C, Carlin JB, Cameron FJ. Age-related pituitary volumes in prepubertal children with normal endocrine function: volumetric magnetic resonance data. J Clin Endocrinol Metab. 2005;90:3274-8.

13. De Sanctis V, Soliman AT, Yassin MA, Di Maio S, Daar S, Elsedfy H, Soliman N, Kattamis C. Hypogonadism in male thalassemia major patients: pathophysiology, diagnosis and treatment. Acta Biomed. 2018;89:6-15.

14. Berkovitch M, Bistritzer T, Milone SD, Perlman K, Kucharczyk W, Olivieri NF. Iron deposition in the anterior pituitary in homozygous beta-thalassemia: MRI evaluation and correlation with gonadal function. J Pediatr Endocrinol Metab. 2000;13:179-84. Erratum in: J Pediatr Endocrinol Metab. 2000;13(8):

15. Shalitin S, Carmi D, Weintrob N, Phillip M, Miskin H, Kornreich L, Zilber R, Yaniv I, Tamary H. Serum ferritin level as a predictor of impaired growth and puberty in thalassemia major patients. Eur J Haematol. 2005;74:93-100.

16. Soliman AT, Khalafallah H, Ashour R. Growth and factors affecting it in thalassemia major. Hemoglobin. 2009;33 Suppl 1:S116-26.

17. Wang ZJ, Wang DJ, Chia JM, Yuan Q, Morriss MC, Rollins NK. A software tool for semi-automated quantification of pituitary volumes. International Society of Magnetic Resonance Imaging in Medicine (ISMRM) 19th annual meeting; 05.2011; Montreal Canada. 2011. P4247.

18. Argyropoulou M, Perignon F, Brunelle F, Brauner R, Rappaport R. Height of normal pituitary gland as a function of age evaluated by magnetic resonance imaging in children. Pediatr Radiol. 1991;21:247-9.

19. Han X, Xiu J, Huang Z, Zhang J, Zhang Z, Dong Y, Yuan X, Liu Q. Three-dimensional magnetic resonance volumetry of the pituitary gland is effective in detecting short stature in children. Exp Ther Med. 2014;8:551-6.

20. Noetzli LJ, Panigrahy A, Hyderi A, Dongelyan A, Coates TD, Wood JC. Pituitary iron and volume imaging in healthy controls. AJNR Am J Neuroradiol. 2012;33:259-65.

21. Ibinaiye PO, Olarinoye-Akorede S, Kajogbola O, Bakari AG. Magnetic Resonance Imaging Determination of Normal Pituitary Gland Dimensions in Zaria, Northwest Nigerian Population. J Clin Imaging Sci. 2015;5:29.

22. Hekmatnia A, Radmard AR, Rahmani AA, Adibi A, Khademi $\mathrm{H}$. Magnetic resonance imaging signal reduction may precede volume loss in the pituitary gland of transfusion-dependent betathalassemic patients. Acta Radiol. 2010;51:71-7.

23. Singer ST, Fischer R, Allen I, Lal A, Vichinsky E, Yuan Q, Wang ZJ. Pituitary iron and factors predictive of fertility status in transfusion dependent thalassemia. Haematologica. 2021;106:1740-4.

24. Takano K, Utsunomiya H, Ono H, Ohfu M, Okazaki M. Normal development of the pituitary gland: assessment with three-dimensional MR volumetry. AJNR Am J Neuroradiol. 1999;20:312-5.

25. Grams AE, Gempt J, Stahl A, Förschler A. Female pituitary size in relation to age and hormonal factors. Neuroendocrinology. 2010;92:128-32.

26. De Sanctis V, Soliman AT, Elsedfy H, Di Maio S, Canatan D, Soliman N, Karimi M, Kattamis C. Gonadal dysfunction in adult male patients with thalassemia major: an update for clinicians caring for thalassemia. Expert Rev Hematol. 2017;10:1095-106.

27. De Sanctis V, Soliman A, Mohamed Y. Reproductive health in young male adults with chronic diseases in childhood. Pediatr Endocrinol Rev. 2013;10:284-96. 
28. Osborn AG. Osborn's brain: imaging, pathology, and anatomy. Salt Lake City: Amirsys; 2013. p. 953.

29. Kessler M, Tenner M, Frey M, Noto R. Pituitary volume in children with growth hormone deficiency, idiopathic short stature and controls. J Pediatr Endocrinol Metab. 2016;29:1195-200. 